\title{
Tissue Expression of Caspase-4 and-5 in Some Chronic Colorectal Diseases \\ ${ }^{1}$ Sumaya H. El-Shazly, ${ }^{2}$ Olfat A. Hammam, ${ }^{1}$ Wafaa M. El-Zefzafy, ${ }^{1}$ Moshira A. Ibrahim \\ ${ }^{1}$ Tropical Medicine - Faculty of Medicine for Girls -Al-Azhar University Cairo, Egypt, \\ ${ }^{2}$ Pathology -Theador Bilharz Research Institute, Cairo, Egypt \\ Corresponding author: Moshira A. Ibrahim,email: drm132.ma@ gmail.com
}

\begin{abstract}
Background: Colonic lining epithelium is subject to variety of insults ranging from inflammatory, infectious to neoplastic processes. One of the most common colon problems are inflammatory bowel disease, colorectal polyps, cancerous growths. Caspases are involved in apoptosis, necrosis, and inflammation. Altered Caspase activity has been associated with a variety of colonic diseases, including inflammatory bowel disease (IBD) and colorectal cancer. Caspases-4 and -5 involvement during intestinal homeostasis and disease has not yet been established.

Aim: Detection of the tissue expression of Caspases-4 and -5 in some chronic colorectal diseases. Methods: 100 patients underwent colonoscopy divided into four groups: colorectal cancer (CRC) (35 patients), inflammatory bowel disease (IBD) (25 patients), colorectal polyp (CRP) (20 patients) and irritable bowel syndrome (IBS) as control (20 patients). The diagnosis was established on the basis of clinical history, laboratory (CBC, ESR, liver function tests, CEA), endoscopic and histological data. Immunohistochemistry (IHC) techniques were employed to examine the cellular expression profile of Caspase-4 and -5 .

Results: Tissue Caspase- 4 and -5 were statistically highly significantly increased in the epithelium of CRC in comparison to the other groups. On the other hand, stromal expression of Caspase -4 and -5 were statistically highly significantly increased in IBD group in comparison to the other groups. Conclusions: This study identified epithelialexpressed Caspases -4 and -5 as biomarkers with diagnostic potential in CRC. Also they were a potential marker of dysplasia in IBD and colorectal polyps.
\end{abstract}

Keywords: Caspase-4 and-5, Colorectal cancer, inflammatory bowel diseases, colorectal polyp.

\section{INTRODUCTION}

The colon is the primary target of many functional and pathological disorders, which may have an acute or chronic presentation. The pattern of colorectal diseases differs in developed countries from developing countries especially in tropical and subtropical areas. Chronic inflammation includes different lesions as polyposis, schistosomiasis and IBD. IBD include ulcerative colitis (UC) and Crohn's disease (CD), which are serious disorders of the GIT involving tissue damage and inflammation leading to bowel impairment. IBD patients have an associated risk of developing (CRC), with a cumulative 30-year risk of UC-associated CRC of approximatly $20 \%{ }^{(1,2)}$.

CRC ranking the 6th cancer in Egypt, representing $4 \%$ of the total cancer cases and $53 \%$ of GIT cancers. Its incidence rates are decreasing for most of the past two decades, which has largely been attributed to development of potential biomarkers that will facilitate the early detection of CRC. Caspases are a family of cysteine proteases with evident roles in cellular homeostasis, apoptosis and inflammation. In humans, Caspases-4 and -5 are the inflammatory Caspases subfamily. They have altered activity in the development of intestinal diseases. Excessive production of the inflammatory cytokines, interleukin (IL)-1b and IL-18, which are dependent upon inflammation-mediated Caspase activation, found in inflammed colons. IL-1b expression is also up- regulated during $\mathrm{CRC}$ and has been identified as a key factor in tumour progression and metastasis ${ }^{(3,4)}$.

Caspase 4 mRNA is found in most tissues examined with the exception of the brain. Highest expression is found in spleen and lung, with moderate expression in heart, intestine and liver. Low expression is observed with skeletal muscle, kidney, and testis .The data suggest that in lung cancer cells, caspase-5 might be a candidate for a tumor suppressor gene. Frame-shift mutations in the caspase- 5 gene have also been revealed in endometrial, colon, and gastric cancers ${ }^{(5,6)}$. The aim of this study was to detect the tissue expression of Caspases- 4 and -5 in some chronic colorectal diseases.

\section{MATERIALS AND METHODS}

The present study was conducted on 100 selected patients with chronic colonic lesions, referred to hepatogastroenterology and infectious disease Department, Alzahraa university Hospital, Cairo, Egypt (from April 2016 to December 2018).

Inclusion criteria: adult and elderly patients with symptoms suggestive of chronic colorectal diseases.

Exclusion criteria: patients with local causes of rectal bleeding as pile and fissures, patients with malignancy other than CRC, patients with suspected toxic megacolon, colonic obstruction, colonic perforation, colectomy or proctocolectomy and 
Tissue Expression of Caspase-4 and-5...

children. Informed consent was obtained from all patients, and the study was approved by the Ethics Committee of hepatogastroenterology and infectious disease Department, Al-Azhar University.

All candidates were subjected to detailed clinical evaluations that include the following:

- Full history taking including symptoms of lower GIT symptoms with stress on: altered bowel habits, abdominal pain, distension, presence of mucous or blood in the stool. Also anorexia, dizziness, palpitation, easy fatigability, loss of weight and pallor.

- Complete clinical examination and P/R examination of the sphincter tone, to exclude any obvious anal or rectal lesions and if the endoscopic preparation has been adequate.

Laboratory investigations: Blood samples were taken for CBC, ESR, liver function tests, PT, INR, CEA, and AFP. Stool analysis: Macroscopically, microscopally and by concentration methods then Occult blood in stool was done by immunohistochemical methods.

Imaging procedures: Conventional Abdominal Ultrasonography and abdominal Computed Tomography (CT) for detection of masses, lymph nodes, metastasis and for staging.

Colonoscopy and colonic biopsy: During the examination, the location, and size of all polypoid lesions, ulcers or masses as well as extension of lesions were noted and described. Polypectomy was done and three biopsies were taken from any abnormal mucosa and masses and these specimens were sent for histopathology.

Histopathological examination: All sections were subjected to light microscopic examination after Hematoxylin and eosin stain for evaluating the histopathological and basic classification of cases according to the etiology. The studied patients were divided according to endoscopical and histopathological examination into the following groups:

Group I: 35 patients with colorectal cancer. They are 19 males (54\%) and 16 females (46\%). Their ages ranged from 22 to 77 years with mean value of $52 \pm 15$ years old.

Group II: 25 patients with inflammatory bowel disease (18 patients were diagnosed as ulcerative colitis and 7 diagnosed as Crohn's). They are (15) males $(60 \%)$ and 10 females $(40 \%)$. Their ages ranged from 18 to 45 years with mean value of $31 \pm 7$ years old.

Group III: 20 patients with colorectal adenomas. They are 13 males $(65 \%)$ and 7 females (35\%). Their ages ranged from 18 to 56 years with mean value of 38 \pm 12 years old

Group IV: 20 patients with symptoms and signs of colorectal diseases and colonoscopic and histopathological examination revealed mild colitis and IBS. They are 15 males (75\%) and 5 females (25\%). Their ages ranged from 20 to 73 years with mean value of $44 \pm 15$ years old.

A diagnosis of IBD was established according to the corresponding criteria. For cases of UC, the true love classification was used to assess severity, for the CD cases; the Crohn's disease activity index was used to assess the disease activity, and the Montreal classification was used to assess the extent of both diseases ${ }^{(7)}$.

Immunohistochemical (IHC) stains: for Caspase-4 and Caspase-5 Antibody, Thermo Fisher Scientific, USA, Catalog \# PA5-20108. This was performed using anti human Caspase- 4 and -5 antibody beside a detection kit DAKO LSAB ${ }^{\circledR}$ System- HRP (DAB, DAKO, Denmark). This technique is an indirect immunohistochemical procedure that uses the antihuman Caspase- 4 and -5 antibodies and horse raddish peroxidase (HRP) with the substrate diaminobenzidine (DAB) as labeling system. All immunostained slides were analyzed using Zeiss microscope with high resolution (Axioscope, Germmany) at power $\mathrm{x} 400$ in 10 successive high power fields. Caspase- 4 and -5 antigens were expressed as brown nuclear staining of both epithelial cells lining glands and inflammatory cells infiltrating stroma. Caspase expression was assessed by two blinded reviewers using a validated semi quantitative scoring method. All IHC stained cells were assessed by a combined score of intensity and percentage of nuclear and cytoplasmic staining of both epithelial and inflammatory cells. Staining intensity was graded using a scale of $0-3$, where $0=$ negative, $1=$ weak, 2 $=$ moderate and $3=$ strong. Positivity Percentage of epithelial or inflammatory cells (amount of stained cells) was graded using a scale of $0-4$, where $0=$ no stained cells, $1=1-25 \%$ stained cells, $2=25-50 \%$ stained cells, $3=50-75 \%$ stained cells and $4=75-$ $100 \%$ stained cells.

Statistical Analysis: Data were collected, reviewed and fed to the computer where statistical analysis was done using the Statistic Package for Social Science version 17 (SPSS 17.0) for windows. Comparing groups was done using Student's t-test. Study of the relationship between variables was done using correlation coefficient. The level of significance was taken at $\mathrm{P}$-value of $<0.05$ " and high significant at $\mathrm{P}$ value of $<0.001$.

\section{RESULTS}

The results and data were collected and analyzed in tables 1-6 and Figures 1- 10. 
Table (1): Age and sex distribution of the studied groups

\begin{tabular}{|c|c|c|c|c|c|c|c|c|}
\hline & \multicolumn{2}{|c|}{$\begin{array}{c}\text { Group I (35) } \\
\text { CRC }\end{array}$} & \multicolumn{2}{|c|}{ Group II (25) IBD } & \multicolumn{2}{|c|}{$\begin{array}{l}\text { Group III (20) } \\
\text { adenomas }\end{array}$} & \multicolumn{2}{|c|}{$\begin{array}{c}\text { Group } \\
\text { IV }(20) \text { Control }\end{array}$} \\
\hline $\begin{array}{c}\text { Age } \\
\text { (years) } \\
\text { (Mean } \pm \\
\text { SD) }\end{array}$ & $\begin{array}{c}52 . \\
11\end{array}$ & $\begin{array}{c} \pm 15 \text {. } \\
37\end{array}$ & $\begin{array}{l}31 . \\
36\end{array}$ & \pm 7.80 & $\begin{array}{c}38 . \\
5\end{array}$ & \pm 12.12 & $\begin{array}{c}44 . \\
6\end{array}$ & \pm 15.63 \\
\hline Sex & $\mathrm{N}$ & $\%$ & $\mathrm{~N}$ & $\%$ & $\mathrm{~N}$ & $\%$ & $\mathrm{~N}$ & $\%$ \\
\hline Female & 16 & 46 & 10 & 40 & 7 & 35 & 5 & 25 \\
\hline Male & 19 & 54 & 15 & 60 & 13 & 65 & 15 & 75 \\
\hline \multicolumn{9}{|c|}{ P-value } \\
\hline & \multicolumn{2}{|c|}{ Group I } & Group I & Group II & \multicolumn{2}{|c|}{ Group 1} & Group I & Group I \\
\hline Age (ye & \multicolumn{2}{|c|}{0.088 (I } & 0.001 & $0.176(\mathrm{~N}$ & \multicolumn{2}{|c|}{$<0.001$} & 0.001 & $0.01(\mathrm{~S}$ \\
\hline Sex & \multicolumn{2}{|c|}{0.287 (I } & $0.698(\mathrm{~N}$ & $1(\mathrm{NS}$ & \multicolumn{2}{|c|}{$0.666(\mathrm{~N}$} & $0.448(1)$ & $0.7(\mathrm{NS}$ \\
\hline
\end{tabular}

HS: highly significant at $p<0.001, \quad$ S: significant at $p=0.05, \quad$ NS: non-significant at $p>0.05$.

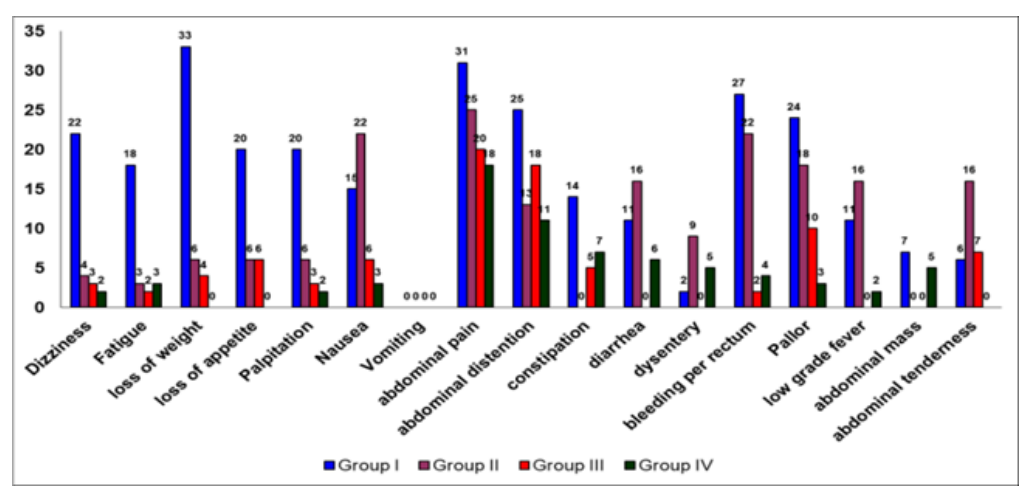

Figure (1): Comparison of the main clinical manifestations among the studied groups

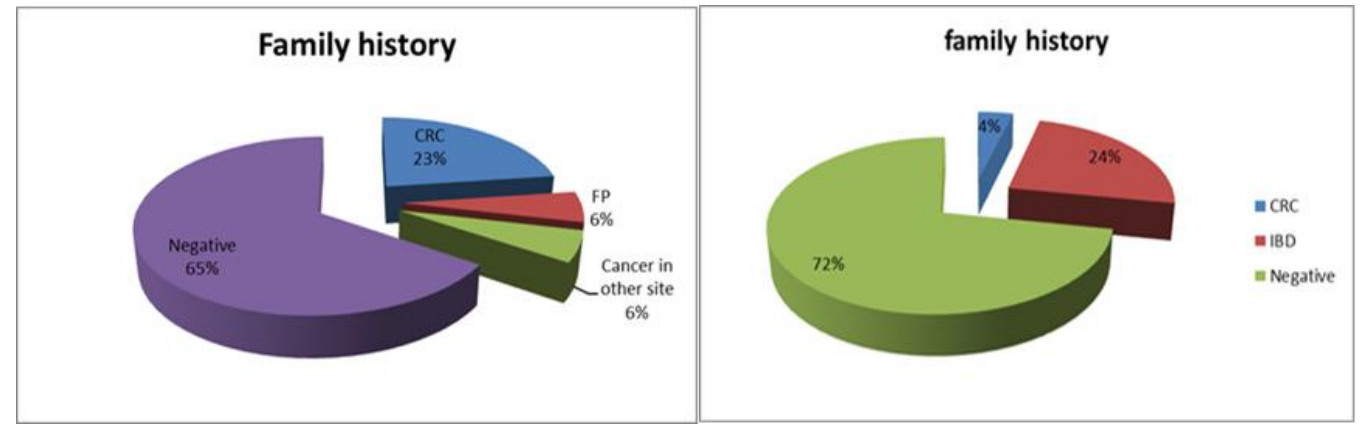

Figure (2): Frequency of family history in group I(left) and II (right) 
Tissue Expression of Caspase-4 and-5...

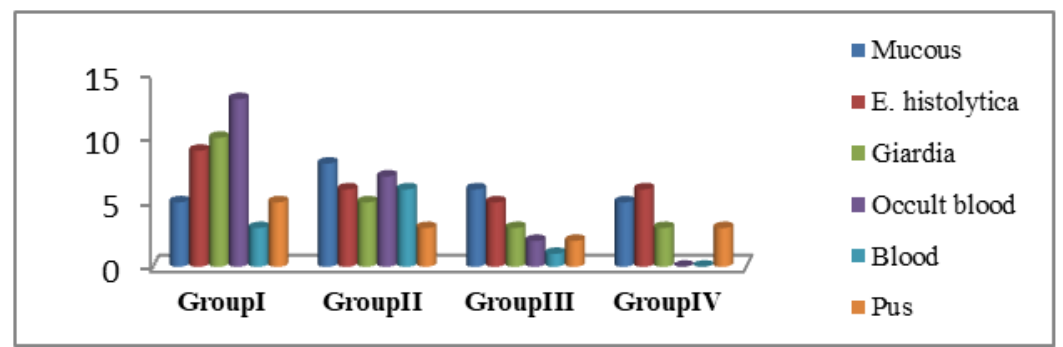

Figure (3): Stool analysis results among the studied groups

Table (2): Comparison of Blood Picture, ESR mean results among the studied groups:

\begin{tabular}{|c|c|c|c|c|c|c|}
\hline & & Group I & & Group II & $\begin{array}{r}\text { Group } \\
\text { III }\end{array}$ & Group IV \\
\hline \multirow{2}{*}{ RBCs $X 10^{12} / \mathrm{L}$} & Mean & 3.96 & & 3.66 & 4.4 & 4.89 \\
\hline & $\pm \mathrm{SD}$ & \pm 0.36 & & \pm 0.37 & \pm 0.34 & \pm 0.38 \\
\hline \multirow{2}{*}{$\mathrm{Hb} \mathrm{gm/dl}$} & Mean & 9.66 & & 9.83 & 12.13 & 13.75 \\
\hline & $\mathrm{SD}$ & \pm 1.43 & & \pm 1.63 & \pm 2.07 & \pm 1.47 \\
\hline \multirow{2}{*}{ MCV (fL) } & Mean & 76.33 & & 79.22 & 87.68 & 90.15 \\
\hline & $\pm \mathrm{SD}$ & \pm 5.8 & & \pm 9.17 & \pm 8.55 & \pm 4.69 \\
\hline \multirow{2}{*}{$\mathrm{MCH}(\mathrm{pg})$} & Mean & 26.69 & & 27.4 & 28.51 & 28.63 \\
\hline & $\pm \mathrm{SD}$ & \pm 2.24 & & \pm 2.57 & \pm 3.4 & \pm 2.13 \\
\hline \multirow{2}{*}{ WBCs $X 10^{9} / \mathrm{L}$} & Mean & 8.35 & & 8.31 & 7.82 & 7.48 \\
\hline & $\pm \mathrm{SD}$ & \pm 2.94 & & \pm 2.51 & \pm 1.05 & \pm 1.81 \\
\hline \multirow{2}{*}{ Plat. X10 10 L } & Mean & 301.1 & & 351.3 & 312.1 & 302.7 \\
\hline & $\pm \mathrm{SD}$ & \pm 18.3 & & \pm 2.24 & \pm 19.1 & \pm 9.3 \\
\hline \multirow{2}{*}{ ESR mm/hr } & Mean & 80.08 & & 91.32 & 15.1 & 8.3 \\
\hline & $\pm \mathrm{SD}$ & \pm 4.35 & & \pm 3.34 & \pm 3.41 & \pm 2.27 \\
\hline \multicolumn{7}{|r|}{ P-value } \\
\hline & $\begin{array}{r}\text { Group } \\
\text { I/IV }\end{array}$ & $\begin{array}{r}\text { Group } \\
\text { II/IV }\end{array}$ & $\begin{array}{c}\text { Group } \\
\text { III/IV }\end{array}$ & $\begin{array}{r}\text { Group } \\
\text { I/II }\end{array}$ & $\begin{array}{r}\text { Grou } \\
\text { p } \\
\text { I/III }\end{array}$ & $\begin{array}{r}\text { Group } \\
\text { II/III }\end{array}$ \\
\hline RBCs X $10^{12} / \mathrm{L}$ & $\begin{array}{r}<0.001(\mathrm{H} \\
\mathrm{S}) \\
\end{array}$ & $\begin{array}{r}<0.001(\mathrm{H} \\
\mathrm{S})\end{array}$ & $0.002(\mathrm{~S})$ & $\begin{array}{r}0.0613(\mathrm{~N} \\
\mathrm{S}) \\
\end{array}$ & $\begin{array}{r}0.011 \\
(\mathrm{~S}) \\
\end{array}$ & $\begin{array}{r}0.001(\mathrm{H} \\
\mathrm{S}) \\
\end{array}$ \\
\hline $\mathrm{Hb} \mathrm{gm} / \mathrm{dl}$ & $\begin{array}{r}<0.001(\mathrm{H} \\
\mathrm{S})\end{array}$ & $\begin{array}{r}<0.001(\mathrm{H} \\
\mathrm{S})\end{array}$ & $\begin{array}{r}0.583(\mathrm{~N} \\
\mathrm{S}) \\
\end{array}$ & $\begin{array}{r}0.479 \\
(\mathrm{NS}) \\
\end{array}$ & $\begin{array}{r}<0.001 \\
(\mathrm{HS}) \\
\end{array}$ & $\begin{array}{r}0.001 \\
(\mathrm{HS})\end{array}$ \\
\hline MCV (fL) & $\begin{array}{r}<0.001(\mathrm{H} \\
\mathrm{S})\end{array}$ & $\begin{array}{r}<0.001(\mathrm{H} \\
\mathrm{S})\end{array}$ & $0.007(\mathrm{~S})$ & $\begin{array}{r}0.192 \\
(\mathrm{NS})\end{array}$ & $\begin{array}{r}<0.001 \\
(\mathrm{HS})\end{array}$ & $0.004(\mathrm{~S})$ \\
\hline $\mathrm{MCH}(\mathrm{pg})$ & $\begin{array}{r}<0.001(\mathrm{H} \\
\mathrm{S}) \\
\end{array}$ & $\begin{array}{r}<0.001(\mathrm{H} \\
\mathrm{S}) \\
\end{array}$ & $\begin{array}{r}0.265(\mathrm{~N} \\
\mathrm{S}) \\
\end{array}$ & $\begin{array}{r}0.785 \\
(\mathrm{NS}) \\
\end{array}$ & $\begin{array}{r}0.001 \\
(\mathrm{HS}) \\
\end{array}$ & $\begin{array}{r}0.001 \\
(\mathrm{HS}) \\
\end{array}$ \\
\hline WBCs X $10^{9} / \mathrm{L}$ & $\begin{array}{r}0.066(\mathrm{NS} \\
\text { ) }\end{array}$ & $\begin{array}{r}0.221(\mathrm{NS} \\
\text { ) }\end{array}$ & $\begin{array}{r}0.899(\mathrm{~N} \\
\mathrm{S})\end{array}$ & $0.44(\mathrm{NS})$ & $\begin{array}{r}0.17(\mathrm{~N} \\
\mathrm{S}) \\
\end{array}$ & $\begin{array}{l}0.48 \\
(\mathrm{NS})\end{array}$ \\
\hline Plat. X109/L & $\begin{array}{r}0.972(\mathrm{NS} \\
\text { ) }\end{array}$ & $0.052(\mathrm{~S})$ & $\begin{array}{r}0.757(\mathrm{~N} \\
\mathrm{S})\end{array}$ & $\begin{array}{r}0.217 \\
(\mathrm{NS})\end{array}$ & $\begin{array}{r}0.813 \\
(\mathrm{NS})\end{array}$ & $\begin{array}{l}0.18 \\
\text { (NS) }\end{array}$ \\
\hline $\mathrm{ESR} \mathrm{mm} / \mathrm{hr}$ & $\begin{array}{r}<0.001(\mathrm{H} \\
\mathrm{S})\end{array}$ & $\begin{array}{r}<0.001(\mathrm{H} \\
\mathrm{S})\end{array}$ & $0.003(\mathrm{~S})$ & $\begin{array}{r}0.177 \\
(\mathrm{NS})\end{array}$ & $\begin{array}{r}<0.001 \\
(\mathrm{HS})\end{array}$ & $\begin{array}{r}<0.001 \\
(\mathrm{HS})\end{array}$ \\
\hline
\end{tabular}

HS: highly significant at $p<0.001, \quad S$ : significant at $p=0.05, \quad$ NS: non-significant at $p>0.05$. 
Table (3): Comparison of liver function tests and tumor marker tests among the studied groups:

\begin{tabular}{|c|c|c|c|c|c|c|}
\hline & & \multicolumn{2}{|c|}{ Group I } & $\begin{array}{c}\text { Group } \\
\text { II }\end{array}$ & $\begin{array}{l}\text { Group } \\
\text { III }\end{array}$ & $\begin{array}{l}\text { Group } \\
\text { IV }\end{array}$ \\
\hline \multirow{2}{*}{ ALT (U/L) } & Mean & \multicolumn{2}{|c|}{25.43} & 20.56 & 17.3 & 18.95 \\
\hline & $\pm \mathrm{SD}$ & \multicolumn{2}{|c|}{ \pm 2.14} & \pm 5.73 & \pm 3.94 & \pm 3.49 \\
\hline \multirow{2}{*}{ AST (U/L) } & Mean & \multicolumn{2}{|c|}{31.74} & 23.56 & 18.1 & 21 \\
\hline & $\pm \mathrm{SD}$ & \multicolumn{2}{|c|}{ \pm 8.80} & \pm 3.65 & \pm 3.57 & \pm 3.37 \\
\hline \multirow{2}{*}{$\begin{array}{c}\text { Bil.T. } \\
\text { (mg/dL) }\end{array}$} & Mean & \multicolumn{2}{|c|}{0.93} & 0.74 & 0.75 & 0.69 \\
\hline & $\pm \mathrm{SD}$ & \multicolumn{2}{|c|}{ \pm 0.02} & \pm 0.15 & \pm 0.03 & \pm 0.13 \\
\hline \multirow{2}{*}{$\mathrm{Alb}(\mathrm{g} / \mathrm{dL})$} & Mean & \multicolumn{2}{|c|}{3.32} & 4.04 & 3.29 & 3.89 \\
\hline & $\pm \mathrm{SD}$ & \multicolumn{2}{|c|}{ \pm 0.5} & \pm 0.405 & \pm 0.169 & \pm 0.32 \\
\hline \multirow{2}{*}{ PT(sec.) } & Mean & \multicolumn{2}{|c|}{14.11} & 12.68 & 13.69 & 12.53 \\
\hline & $\pm \mathrm{SD}$ & \multicolumn{2}{|c|}{ \pm 2.12} & \pm 0.80 & \pm 0.72 & \pm 1.38 \\
\hline \multirow{2}{*}{ INR } & Mean & \multicolumn{2}{|c|}{1.42} & 1.26 & 1.21 & 1.21 \\
\hline & $\pm \mathrm{SD}$ & \multicolumn{2}{|c|}{ \pm 0.34} & \pm 0.18 & \pm 0.17 & \pm 0.13 \\
\hline \multirow{2}{*}{$\begin{array}{c}\mathrm{AFP}(\mathrm{ng} / \mathrm{ml} \\
)\end{array}$} & Mean & \multicolumn{2}{|c|}{28.83} & 24.56 & 29.2 & 1.6 \\
\hline & $\pm \mathrm{SD}$ & \multicolumn{2}{|c|}{ \pm 5.75} & \pm 3.29 & \pm 5.25 & \pm 0.09 \\
\hline \multirow{2}{*}{$\begin{array}{l}\text { s.CEA } \\
\text { (ng/ml) }\end{array}$} & Mean & \multicolumn{2}{|c|}{30.38} & 3.17 & 2.68 & 1.44 \\
\hline & $\pm \mathrm{SD}$ & \multicolumn{2}{|c|}{ \pm 9.32} & \pm 1.19 & \pm 0.35 & \pm 0.21 \\
\hline \multicolumn{7}{|c|}{ P-value } \\
\hline & $\begin{array}{l}\text { Group } \\
\text { I/IV }\end{array}$ & $\begin{array}{l}\text { Group } \\
\text { II/IV }\end{array}$ & $\begin{array}{l}\text { Group } \\
\text { III/IV }\end{array}$ & $\begin{array}{l}\text { Group } \\
\text { I/II }\end{array}$ & $\begin{array}{c}\text { Group } \\
\text { I/III }\end{array}$ & $\begin{array}{c}\text { Group } \\
\text { II/III }\end{array}$ \\
\hline ALT (U/L) & $0.029(\mathrm{~S})$ & $\begin{array}{c}0.346 \\
(\mathrm{NS})\end{array}$ & $\begin{array}{l}0.367 \\
(\mathrm{NS})\end{array}$ & $0.068(\mathrm{~S})$ & $0.007(\mathrm{~S})$ & $0.07(\mathrm{NS})$ \\
\hline AST (U/L) & $\begin{array}{c}0.107 \\
\text { (NS) }\end{array}$ & $0.18(\mathrm{NS})$ & $\begin{array}{c}0.134 \\
(\mathrm{NS})\end{array}$ & $\begin{array}{c}0.168 \\
\text { (NS) }\end{array}$ & $0.042(\mathrm{~S})$ & $0.004(\mathrm{~S})$ \\
\hline $\begin{array}{c}\text { Bil.T. } \\
\text { (mg/dL) }\end{array}$ & $\begin{array}{l}0.09 \\
\text { (NS) }\end{array}$ & $\begin{array}{c}0.188 \\
(\mathrm{NS})\end{array}$ & $0.28(\mathrm{NS})$ & $\begin{array}{l}0.146 \\
\text { (NS) }\end{array}$ & $\begin{array}{l}0.223 \\
\text { (NS) }\end{array}$ & $0.88(\mathrm{NS})$ \\
\hline $\mathrm{Alb}(\mathrm{g} / \mathrm{dL})$ & $\begin{array}{l}<0.001 \\
(\mathrm{HS})\end{array}$ & $\begin{array}{c}0.182 \\
\text { (NS) }\end{array}$ & $\begin{array}{c}<0.001 \\
(\mathrm{HS})\end{array}$ & $\begin{array}{l}<0.001 \\
(\mathrm{HS})\end{array}$ & $\begin{array}{l}0.745 \\
\text { (NS) }\end{array}$ & $\begin{array}{l}0.001 \\
\text { (HS) }\end{array}$ \\
\hline PT(sec.) & $0.004(\mathrm{~S})$ & $\begin{array}{c}0.651 \\
(\mathrm{NS})\end{array}$ & $0.002(\mathrm{~S})$ & $\begin{array}{c}0.002 \\
(\mathrm{~S})\end{array}$ & $0.4(\mathrm{NS})$ & $\begin{array}{c}0.001 \\
\text { (HS) }\end{array}$ \\
\hline INR & $0.008(\mathrm{~S})$ & $\begin{array}{l}0.227 \\
(\mathrm{NS})\end{array}$ & $\begin{array}{l}0.915 \\
(\mathrm{NS})\end{array}$ & $0.047(\mathrm{~S})$ & $0.012(\mathrm{~S})$ & $0.31(\mathrm{NS})$ \\
\hline$\underset{)}{\mathrm{AFP}(\mathrm{ng} / \mathrm{ml}}$ & $\begin{array}{c}<0.001 \\
(\mathrm{HS})\end{array}$ & $\begin{array}{c}<0.001 \\
(\mathrm{HS})\end{array}$ & $\begin{array}{c}<0.001 \\
(\mathrm{HS})\end{array}$ & $\begin{array}{l}0.26 \\
(\mathrm{NS})\end{array}$ & $\begin{array}{c}0.721 \\
(\mathrm{NS})\end{array}$ & $0.53(\mathrm{NS})$ \\
\hline $\begin{array}{l}\text { s.CEA } \\
\text { (ng/ml) }\end{array}$ & $\begin{array}{l}<0.001 \\
(\mathrm{HS})\end{array}$ & $\begin{array}{c}0.221 \\
(\mathrm{NS}) \\
\end{array}$ & $\begin{array}{c}0.389 \\
\text { (NS) } \\
\end{array}$ & $\begin{array}{l}<0.001 \\
(\mathrm{HS})\end{array}$ & $\begin{array}{c}<0.001 \\
(\mathrm{HS})\end{array}$ & $\begin{array}{l}0.797 \\
\text { (NS) }\end{array}$ \\
\hline
\end{tabular}

HS: highly significant at $p<0.001, \quad$ S: significant at $p=0.05, \quad$ NS: non-significant at $p>0.05$. 
Tissue Expression of Caspase-4 and-5...

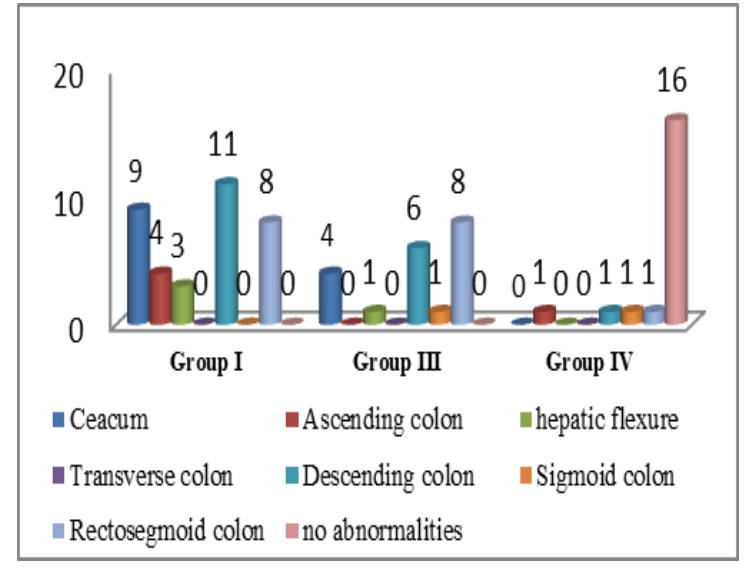

Figure (4): Frequency and location of lesions among the studied groups (I, III and IV)

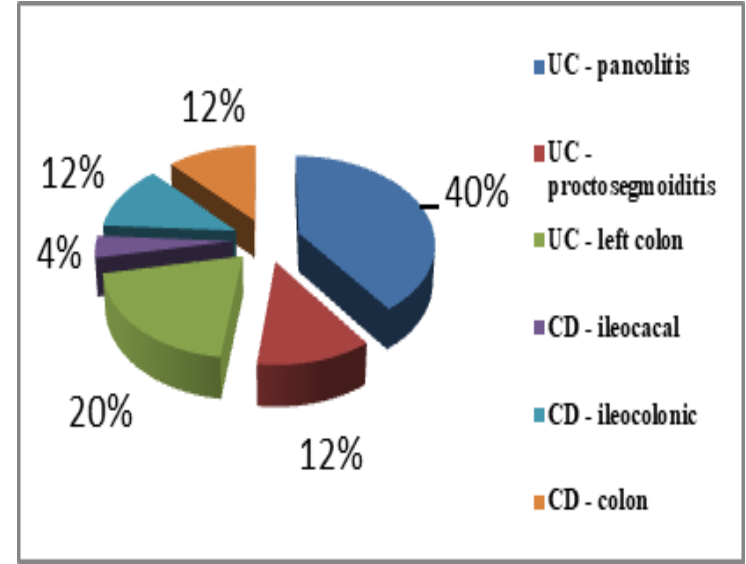

Figure (5): Frequency and location of lesions in group II

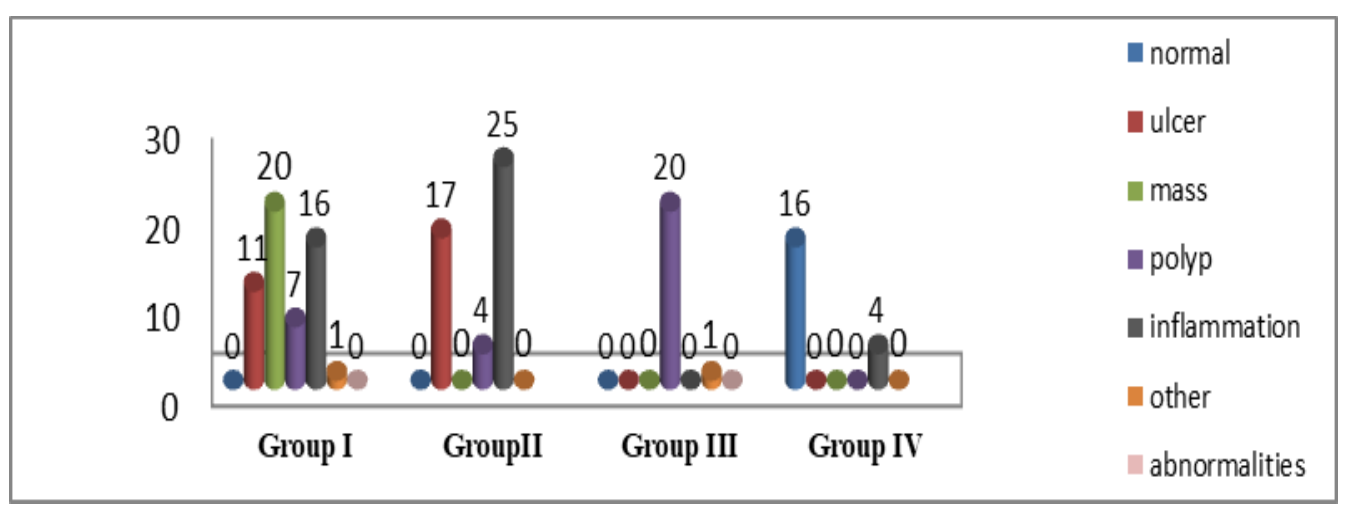

Figure (6): Frequency of different endoscopic findings among the studied groups
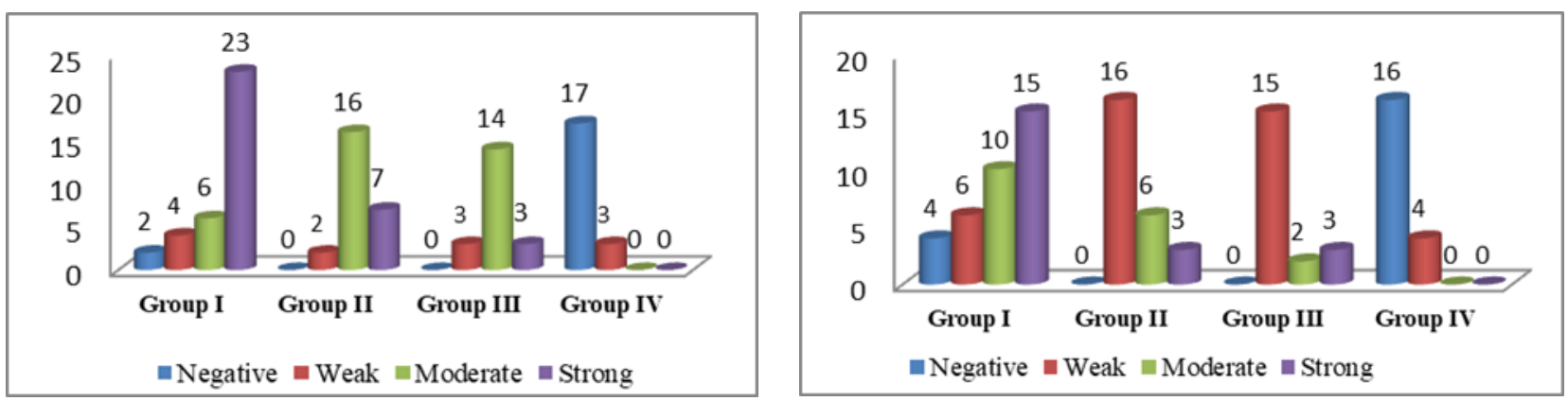

Our results showed statistically significant increase of

both ECLG Caspase-4 and -5 intensity of expression and expression level in all groups in comparison with control group and in CRC group in comparison with other groups. At the level of lamina propria (stroma), there was statistically highly significant increase of SIC level of expression of Caspase -4 in all groups in comparison with control group and in IBD group in comparison with all groups. While there was statistically highly significant increase of SIC level of expression of Caspase -5 in all groups in comparison with control group and in CRC group in comparison with CRP group and in IBD group in comparison with all groups.

Figure (7-8): Expression of Caspase- 4 (left) and Caspase-5 (right) and scoring of stained ECLG among the studied groups. ECLG: epithelial cells lining glands. 


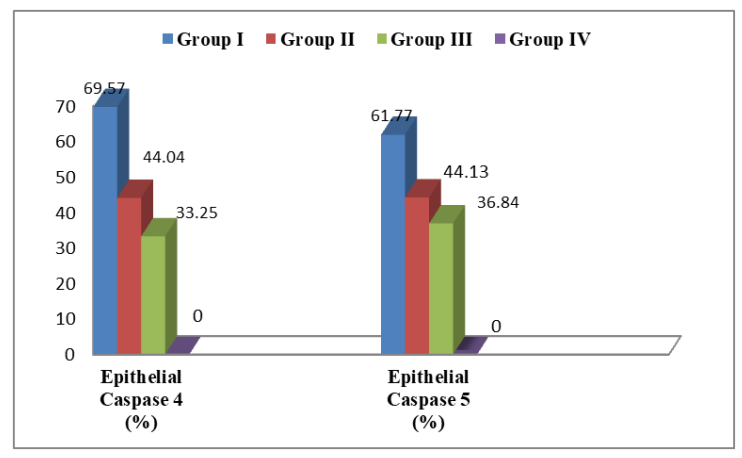

Figure (9): Comparison of epithelial expression level of Caspase -4 and Caspase -5 in the studied groups

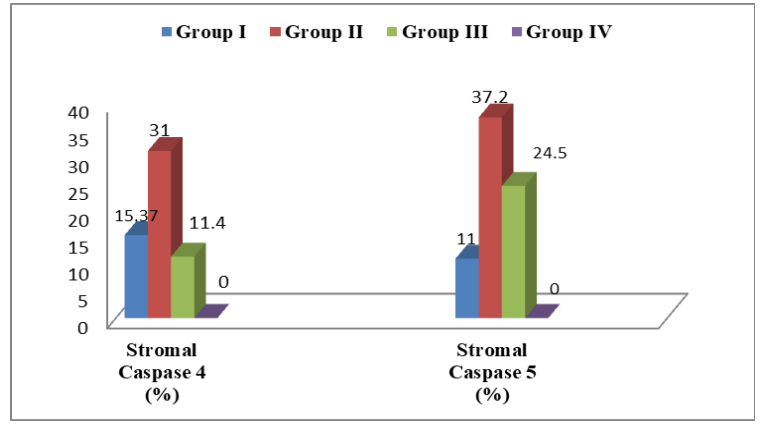

Figure (10): Comparison of SIC level of expression of expression of Caspase -4 and Caspase -5 in the studied groups. SIC; stromal inflammatory cells

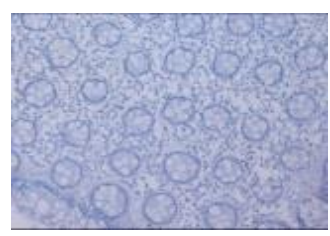

(a)

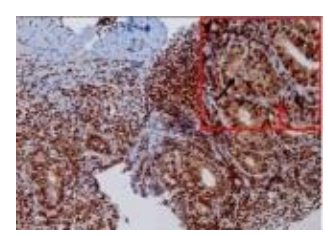

(b)

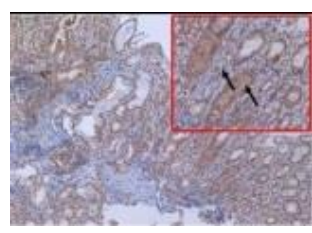

(c)

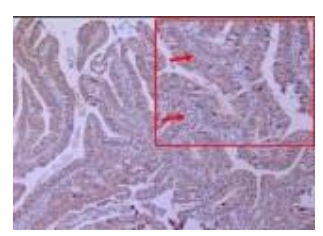

(d)

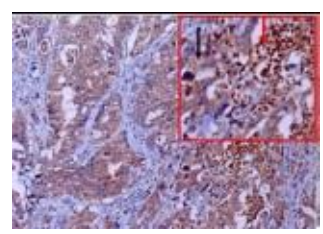

(e)

Figure (11): Caspase-4 IHC (a) Control: case of mild colitis, showing negative expression of caspase 4 (IHC, $\mathrm{DAB}, \mathrm{x} 200)$. (b) Ulcerative Colitis case, showing moderate to marked expression of caspase 4 immunostain in the cytoplasm of cells lining the colonic glands (black arrow) and moderate expression of caspase 4 in inflammatory cells (red arrow) (IHC, DAB, X200). (c) A case of Crohns Disease, showing moderate expression of caspase 4 immunostain in the cytoplasm of cells lining the colonic glands (black arrow) (H \& E X200). (d) A cases of tubulovillous adenoma, showing moderate expression of caspase 4 immunostain in the cytoplasm of cells lining the colonic glands (red arrow) (H \& E X40). (e) A cases of moderately differentiated adenocarcinoma, showing marked expression of caspase 4 immunostain in the cytoplasm of cells lining the colonic glands (black arrow) and positive staining of inflammatory cells (red arrow) (H\&E X200).

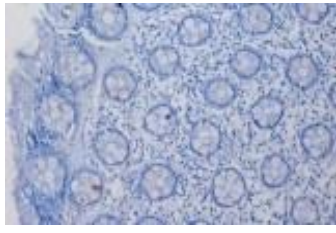

(a)

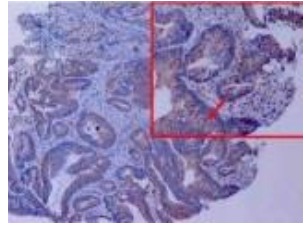

(b)

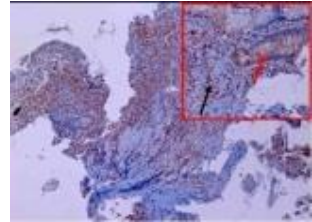

(c)

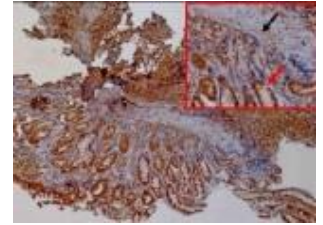

(d)

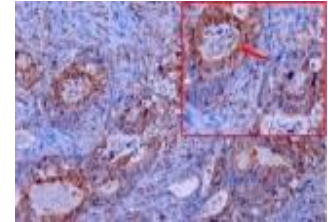

(e)

Figure (12): Caspase-5 IHC: (a) Control case of mild colitis, showing negative expression of caspase 5 (IHC, $\mathrm{DAB}, \mathrm{x} 200$ ); (b) Ulcerative Colitis case, showing mild to moderate expression of caspase 5 immunostain in the cytoplasm of cells lining the colonic glands (red arrow) and positive staining of inflammatory cells (black arrow) (IHC, DAB, x200) (IHC, X100). (c) A case of Crohns Disease, showing moderate expression of caspase 5 immunostain in the cytoplasm of cells lining the colonic glands (red arrow) and positive staining of inflammatory cells (black arrow) (H \& E X100). (d) A cases of tubulovillous adenoma, showing moderate expression of caspase 5 immunostain in the cytoplasm of cells lining the colonic glands (red arrow) (H \& E X40). (e) A cases of moderately differentiated adenocarcinoma, showing moderate to marked expression of caspase 5 immunostain in the cytoplasm of cells lining the colonic glands (red arrow) ( H \& E X100). 
Table (4): Correlation Significance (r) among Caspase 4 and 5 parameters and the main laboratory tests results:

\begin{tabular}{|c|c|c|c|c|c|}
\hline & & $\begin{array}{c}\text { Caspase } 4 \\
\text { epithelial } \\
\text { color } \\
\text { intensity } \\
\end{array}$ & $\begin{array}{c}\text { Caspase } 4 \\
\text { epithelial cell } \\
\text { level }\end{array}$ & $\begin{array}{c}\text { Caspase } 5 \\
\text { epithelial color } \\
\text { intensity }\end{array}$ & $\begin{array}{c}\text { Caspase } 5 \\
\text { epithelial cell } \\
\text { level }\end{array}$ \\
\hline \multirow{2}{*}{$\begin{array}{c}\text { RBCs X } \\
1012 / L\end{array}$} & $(\mathbf{r})$ & $\mathbf{0 . 3 2 3 5}$ & 0.449 & $\mathbf{0 . 1 0 9 3}$ & -0.44 \\
\hline & P.value & 0.001 (HS) & $<0.001(\mathrm{HS})$ & 0.279 (NS) & $<0.001($ HS) \\
\hline \multirow{2}{*}{$\begin{array}{c}\text { WBCs X } \\
\text { 109/L }\end{array}$} & $(\mathbf{r})$ & 0.0036 & 0.176 & 0.0024 & 0.19 \\
\hline & P.value & $\begin{array}{c}0.972 \\
\text { (NS) }\end{array}$ & $0.081(\mathrm{NS})$ & 0.981 (NS) & 0.07(NS) \\
\hline \multirow{2}{*}{ Hb gm/dL } & $(\mathbf{r})$ & 0.4101 & 0.545 & 0.0849 & -0.50 \\
\hline & P.value & $<0.001(\mathrm{HS}$ & $<0.001(\mathrm{HS})$ & 0.401 (NS) & $<0.001($ HS $)$ \\
\hline \multirow{2}{*}{ Plat. X109/L } & $(\mathbf{r})$ & $\mathbf{0 . 0 0 8 2}$ & 0.203 & 0.1510 & 0.24 \\
\hline & P.value & 0.935 (NS) & $0.104(\mathrm{NS})$ & 0.134 (NS) & $0.06(\mathrm{NS})$ \\
\hline \multirow{2}{*}{ ESR mm/hr } & $(\mathbf{r})$ & 0.4742 & 0.62 & 0.1818 & 0.53 \\
\hline & P.value & $<0.001$ (HS & $<0.001($ HS $)$ & 0.070(NS) & $<0.001(\mathrm{HS})$ \\
\hline \multirow{2}{*}{ ALT U/L } & $(\mathbf{r})$ & $\mathbf{0 . 1 1 8 6}$ & 0.165 & 0.0151 & 0.326 \\
\hline & P.value & $0.240(\mathrm{NS})$ & $0.102(\mathrm{NS})$ & 0.881 (NS) & $0.001(\mathrm{HS})$ \\
\hline \multirow[t]{2}{*}{ AST U/L } & $(\mathbf{r})$ & 0.111 & 0.237 & 0.0273 & 0.159 \\
\hline & P.value & 0.270 (NS) & 0.017(NS) & $0.788(\mathrm{NS})$ & $0.115(\mathrm{NS})$ \\
\hline \multirow{2}{*}{ Bil.T. mg/dI } & $(\mathbf{r})$ & 0.0202 & 0.156 & $\mathbf{0 . 1 4 3 7}$ & 0.253 \\
\hline & P.value & $0.842(\mathrm{NS})$ & $0.121(\mathrm{NS})$ & $0.154(\mathrm{NS})$ & $0.011(\mathrm{~S})$ \\
\hline \multirow{2}{*}{ Alb g/dL } & $(\mathbf{r})$ & 0.2421 & 0.287 & 0.1356 & 0.285 \\
\hline & P.value & $0.015(\mathrm{~S})$ & $0.004(\mathrm{~S})$ & 0.179(NS) & $0.004(\mathrm{~S})$ \\
\hline \multirow{2}{*}{ AFP } & $(\mathbf{r})$ & 0.1719 & 0.217 & 0.1641 & 0.273 \\
\hline & P.value & 0.087(NS) & $0.030(S)$ & $0.103(\mathrm{NS})$ & $0.006(\mathrm{~S})$ \\
\hline \multirow{2}{*}{ PT } & $(\mathbf{r})$ & 0.1690 & 0.185 & 0.0031 & 0.325 \\
\hline & P.value & $0.093(\mathrm{NS})$ & $0.066(\mathrm{NS})$ & $0.975(\mathrm{NS})$ & $0.001(\mathrm{HS})$ \\
\hline \multirow{2}{*}{ s.CEA } & $(\mathbf{r})$ & 0.355 & 0.521 & 0.405 & 0.390 \\
\hline & P.value & $<0.001(\mathrm{HS})$ & $<0.001($ HS) & $<0.001(\mathrm{HS})$ & $<0.001($ HS) \\
\hline
\end{tabular}

HS: highly significant at $\mathrm{p}<0.001, \quad$ S: significant at $\mathrm{p}=0.05, \quad$ NS: non-significant at $\mathrm{p}>0.05$.

Table (5): Correlation Significance (r) among Caspase 4 and 5 parameters and the endoscopic grades of IBD:

\begin{tabular}{|c|c|c|c|c|c|c|c|c|}
\hline \multirow{3}{*}{$\begin{array}{c}\text { Endoscopic } \\
\text { grades of } \\
\text { IBD }\end{array}$} & \multicolumn{2}{|c|}{$\begin{array}{c}\text { spase } 4 \text { epithelial color } \\
\text { intensity }\end{array}$} & \multicolumn{2}{|c|}{$\begin{array}{c}\text { pase } 4 \text { epithelial cell } \\
\text { level }\end{array}$} & \multicolumn{2}{|c|}{$\begin{array}{c}\text { pase } 5 \text { epithelial color } \\
\text { intensity }\end{array}$} & \multicolumn{2}{|c|}{$\begin{array}{c}\text { pase } 5 \text { epithelial cell } \\
\text { level }\end{array}$} \\
\hline & (r) & P. value & $(\mathbf{r})$ & P. value & $(\mathbf{r})$ & P. value & $(\mathbf{r})$ & P. value \\
\hline & 0.74 & $<0.001(\mathrm{HS})$ & 0.22 & $0.30(\mathrm{NS})$ & 0.260 & $0.21(\mathrm{NS})$ & 0.05 & $0.81(\mathrm{NS})$ \\
\hline
\end{tabular}

Table (6 ): Correlation Significance (r) among Caspase 4 and 5 parameters and the pathological grades of adenocarcinoma:

\begin{tabular}{|c|c|c|c|c|c|c|c|c|}
\hline \multirow{3}{*}{$\begin{array}{l}\text { Histopathological } \\
\text { grades of } \\
\text { adenocarcinoma }\end{array}$} & \multicolumn{2}{|c|}{$\begin{array}{c}\text { Caspase } 4 \\
\text { epithelial color } \\
\text { intensity }\end{array}$} & \multicolumn{2}{|c|}{$\begin{array}{l}\text { Caspase } 4 \text { epithelial } \\
\text { cell level }\end{array}$} & \multicolumn{2}{|c|}{$\begin{array}{l}\text { Caspase } 5 \text { epithelia } \\
\text { color intensity }\end{array}$} & \multicolumn{2}{|c|}{$\begin{array}{l}\text { Caspase } 5 \text { epithelia } \\
\text { cell level }\end{array}$} \\
\hline & $(\mathbf{r})$ & P. value & (r) & e & (r) & $\mathbf{P}$. & $(\mathbf{r})$ & Iue \\
\hline & 0.13 & $0.46(\mathrm{NS})$ & 0.194 & $0.26(\mathrm{NS})$ & 0.161 & $0.35(\mathrm{NS})$ & 0.023 & 0.89 (NS) \\
\hline
\end{tabular}




\section{DISCUSSION}

In Egypt, CRC is the third most common tumor in males and females where it ranks the fifth. Recent interest in Egyptian CRC has been raised when personal observations and epidemiologic studies revealed a high incidence of the disease among the young Egyptian population ${ }^{(8)}$.

There are no defined clinical features to predict progression from inflammation to low-grade dysplasia, and onto advanced neoplasia. Therefore, histological assessment of biopsy tissue and the identification of dysplasia are relied upon heavily when making decisions regarding the management and the associated risk of CRC development, and when there are challenges with the decision of the requirement for colectomy in IBD. However, there are a number of issues associated with this current CRC surveillance system: first, the difficulty in identification of true dysplasia versus chronic colonic inflammation. Secondly, patients undergoing regular colonoscopic surveillance can develop CRC without prior dysplasia. Therefore, there is a serious requirement for identifiers of neoplasia which can be assessed in parallel with tissue histology for the identification of CRC ${ }^{(9)}$. Therefore, we studied the altered expression of caspasae- 4 and -5 in some chronic colonic lesions including colorectal polyps, IBD and CRC.

Epidemiological evidence indicates that a family history of colorectal cancer (CRC) is associated with a higher personal CRC risk. The mechanisms underlying familial clustering of CRC remain largely unknown. It may be heritable predisposition to epigenomic instability and the development of tumors with hypomethylation. In contrast, this study showed that $65 \%$ of patients in CRC group had no family history of malignancy and $23 \%$ had family history of malignancy. This is in acceptance with Dabbous et al. $(\mathbf{1 0}, 11)$

Also regarding IBD group in our study, only $24 \%$ of patients had family history of inflammatory bowel disease (IBD). This is in agreement with Esmat et al. (7) who explained this as the under diagnosis of these diseases due to a low disease awareness; confusion of IBD with other causes of infectious diarrhoea and limited access to diagnostic tools as a result of the limited resources available in community health centers.

Regarding laboratory results in the present work, there was high incidence of iron deficiency anemia in both CRC and IBD groups in comparison with the other two groups. This is an evident of statistically highly significant decrease of the RBC and HB mean level and increase of $\mathrm{MC}$ and $\mathrm{MCH}$ mean level. This is in acceptance with Dabbous et al. ${ }^{(11)}$ who concluded that anemia is the most clinically valuable predictive tool for CRC. Regarding IBD group, Kaitha et al. ${ }^{(12)}$ and Patel et al. ${ }^{(13)}$ reported that anemia is the most common complication as well as an extra intestinal manifestation of inflammatory bowel disease (IBD).

There was obvious increase of the mean level of ESR in CRC and IBD groups with statistically high significance in comparison with CRP and control group. This comes in agreement with Mohamed et al. (14) and Ananthakrishnan et al. ${ }^{(15)}$ who reported that elevated level of ESR is associated with increased risk of CRC in patients with IBD.

In the current work, there was statistically highly significant increase of CEA mean value in CRC group in comparison with other groups. This is in agreement with Vukobrat-Bijedic et al. ${ }^{(16)}$ who stated that CEA is one of the most common tumor markers for colorectal cancer that are currently utilized clinically.

In CRC group, the left sided tumor is more frequent in our study (54\%) than right sided tumor (46\%), while in CRP group the lesions were more frequent in rectosegmoid colon. This agrees with Yeo et al. ${ }^{(17)}$. In contrast with Metwally et al. ${ }^{(18)}$ who concluded that right colon represent the commonest site. In IBD group, pancolitis was the most prevalent lesion in UC patients, while ileocolonic and colonic were the most prevalent in Crohn's disease patients. This agrees with Maher and Nassar ${ }^{(19)}$ and Moustafa et al. ${ }^{(20)}$. In contrast with Mohamed et al. ${ }^{(14)}$ and Esmat et al. ${ }^{(7)}$ who found that left-sided colitis was more prevalent.

Our study revealed that the main endoscopic picture in CRC group was mass in $(57 \%)$ and malignant ulcer in $(31 \%)$ and histopathology revealed grade -2 adenocarcinoma in $80 \%$ of patients. Also, Dabbous et al. ${ }^{(11)}$ reported in their study that Perrectum examination (BPR) revealed masses in $35 \%$ of CRC patients, while all CRC patients had either rectal or colonic masses. This is in agreement with us and Yeo et al. ${ }^{(17) .}$ However, Metwally et al. ${ }^{(18)}$ reported that pos-toperative pathology showed classic adenocarcinoma in $64.2 \%$ of cases, and about $53 \%$ of cases were of moderate grade.

In IBD group, the endoscopic examination revealed severe inflammation in all patients associated with ulcers in $68 \%$ of patients. Ulcerative colitis was moderate in $36 \%$ of patients while Crohn's disease was Grade- 2 in $12 \%$ of patients. This was in consistence with Esmat et al. ${ }^{(7)}$.

In CRP group, the endoscopic examination revealed polyp in all patients, $75 \%$ of them in left 
colon. Histopathological examination of polyps revealed hyperplastic polyps in $50 \%$ of patients. The endoscopic examination of the control group was normal apart from mild colitis in 4 patients (20\%). Dabbous et al. ${ }^{(11)}$ reported that all CRP patients had polyps, $30 \%$ of them were hyperplastic polyp.

There was statistically highly significant increase of the expression pattern (color intensity) of Caspases4 and -5 in epithelial cells (lining glands) in CRC group in comparison with other groups. Also, in all groups in comparison with control group. When we examined the level of epithelial expression in the different groups we found that there was statistically highly significant increase of ECLG level of expression of Caspase- 4 and -5 in CRC group in comparison with other groups.

Regarding the IBD group, there was statistically highly significant increase in ECLG level of expression of Caspase- 4 in comparison with CRP and control groups, and of Caspase-5 in comparison with the control group only. This is in agreement with Flood et al. ${ }^{(4)}$ who concluded that Caspases-4 and -5 expression was elevated significantly in neoplastic tissue as well as in inflammed colonic tissue. Taken together, these data support the hypothesis declaring that inflammatory Caspases- 4 and-5 have an important role in intestinal inflammation observed during IBD, although the mechanistic details for these observations are still underway.

Another significant observation from our study, was that tissue staining for Caspases- 4 and-5 in neoplastic tissues was even stronger than that observed in inflamed tissue while epithelial cells showed strongly positive for Caspases-4 and -5 , suggesting that inflammatory cells may have a role in inducing the expression of Caspases-4 and -5 in colon carcinomas.

Flood et al. ${ }^{(4)}$ reported that epithelial expression level of Caspases-4 and -5 were still restricted dramatically to neoplastic tissue, even within areas of severely inflamed tissue. Areas of normal and inflamed tissue from CRC patient, IBD and control patients' resections, all remained completely negative for epithelial Caspases-4 and -5 expression. Although, a marked increase in Caspases -4 and -5 positively stained intraepithelial infiltrating lymphocytes and macrophages was observed in areas of inflamed/dysplastic tissue. Supporting our observations, Davis et al. ${ }^{(21)}$ stated that in IBD group, intraepithelial cells inflammatory Caspases-4 and -5 were found to be significantly elevated, suggesting that pyroptosis may be involved in the development of IBD. Moreover therapeutic drugs for IBD such as mesalamine and corticosteroids may relate to the inhibition of pyroptosis in IEC (intraepithelial cells).

A study of Mathonnet et al. (22) using cotransplanted endothelial colony forming and mesenchymal stem cells revealed a role for Caspase- 4 expression in neovasculogenesis. Exposure of epithelial cells, or their precursor intestinal stem cells, to proinflammatory cytokines can induce cancer stem cell (CSC) markers and cause tumour formation in vivo. This may represent a plausible mechanism for the elevated Caspases-4 and -5 expressions in neoplastic epithelial cells. Although, epithelial cells (lining glands) (ECLG) level of expression of Caspase-4 and -5 was higher than stromal inflammatory cells (SIC) level of expression of Caspase- 4 and -5 , the expression of Caspases within the stromal inflammatory cells showed statistically highly significant increase in IBD group in comparison with the other groups. While Flood et al. (4) study demonstrated that inflammatory Caspases were expressed highly by infiltrating immune cells in areas of active inflammation in the gastrointestinal tract of IBD Patients.

In addition, there was statistically highly significant increase of the expression of Caspases within the stromal inflammatory cells in CRC group in comparison with control group in Caspase-4 and in comparison with CRP and control groups in Caspase5. This is in agreement with Flood et al. ${ }^{(4)}$ who reported that there was a more marked increase in Caspases- 4 and -5 expression levels within the lamina propria of tumour tissue compared to adjacent normal tissue, reflecting the enhanced inflammation occurring within the local tumour microenvironment.

Alternatively, there may be different, unrelated mechanisms responsible for Caspases-4 and -5 expressions in inflammatory cells and epithelial cells, particularly as expression in neoplastic epithelium that was observed in both IBD and sporadic CRC tissue. Expression of Caspases-4 and -5 within epithelial cells might have a difference from that within infiltrating immune cells of the stroma. Previous analysis of the role of nuclear factor-kappa B (NF-jB) in both epithelial and infiltrating myeloid cells during a murine model of colitis-associated CRC revealed that activation of this inflammatory transcription factor in the two different cell types drives carcinogenesis through distinct mechanisms, involving stimulation of proinflammatory cytokines by infiltrating myeloid cells and prevention of intestinal epithelial cell (IEC) death with tumorigenic potential. Caspase-4 has been attributed previously with a role in lipopolysaccharide 
(LPS)-mediated NF-jB activation. Thus, Caspases-4 and -5 may be responsible for driving tumour initiation by preventing the death of IECs during CRC ${ }^{(23)}$.

Shibamoto et al. ${ }^{(24)}$ demonstrated that Caspase-4 expression, which may contribute to the early phase of gastrointestinal carcinoma progression and lymphatic invasion. Therefore, this may predict not only the prognosis, but also early phase tumor progression. In addition, $\mathbf{L u}$ et al. ${ }^{(25)}$ reported that inflammation is an essential driving force in the development of epithelial originated tumours, and their observation of an increased infiltration of Caspases- 4 and -5 -expressing immune cells to the developing tumour site may serve to establish the tumour inflammatory microenvironment. In agreement with our results, Smyth et al. ${ }^{(26)}$ concluded that instead of repressing tumour growth, inflammatory cells can promote tumour growth, particularly during inflammationassociated cancer.

Regarding CRP group, there was statistically highly significant increase of the expression level of Caspase- 4 and-5 within the (ECLG) and the stromal inflammatory cells in comparison with control group. This is in consistence with Mc Skeane et al. ${ }^{(27)}$ who concluded that Caspase- 4 and -5 was highly expressed in the epithelium of all four types of colorectal polyps with dysplasia, with a significant increase in the level of expression from LGD to HGD ( $\mathrm{p}<0.01)$. Similarly, stromal expression also increased in relation to the degree of dysplasia. In our study, we did not classify as all patients were only with low grade dysplasia. Moreover Flood et al. ${ }^{(4)}$ in an attempt to determine the neoplastic stage at which the switch to epithelial expression of Caspases- 4 and -5 occurs, normal and dysplastic areas of polyp tissue were stained and scored for Caspase-4. All areas of normal mucosa were void of epithelial Caspase-4 expression. Four of the five dysplastic polyp tissues examined were positive for epithelial Caspase-4, while one remained negative, suggesting that the switch to epithelial expression may occur during low-grade dysplasia.

There was statistically highly significant correlations between Caspase4 epithelial cell expression level and RBC, HB (negative correlations), ESR and CEA (positive correlation). While, there was statistically highly significant correlations between Caspase 5 epithelial cell level and RBC, HB (negative correlations), ESR, ALT, PT and CEA (positive correlation). These observations in our study indicated good correlation between Caspases -4 and -5 and the natural progression of disease specially in CRC group. Anemia, which was chief manifestation in low immunity groups, ESR and CEA, which are known markers for malignancy and autoimmune aggregation.

There was statistically highly significant correlations between Caspase4 epithelial color intensity and endoscopic grades of IBD. On the other hand, there was no statistically significant correlations between either Caspase 4 or 5 and the pathological grade adenocarcinoma. Oficjalska et al. ${ }^{(28)}$ reported a strong correlation between inflammation score and Caspases-4 expression in UC biopsies, which supports the hypothesis showing that Caspases- 4 contribute to intestinal inflammation, most probably through activation of the non-canonical inflammasome, leading to increased IL-1b and IL-18 production . Flood et al. ${ }^{(4)}$ reported that expression of Caspase-4 in the lamina propria correlated clearly with the extent of inflammation and Mayo score with maximum Caspase-4 expression present in sections with inflammatory and Mayo scores from moderate to strong (scores of 2 and 3). Caspase-5 IHC (immunohistochemistry) staining intensity and percentage expression within the lamina propria correlated with inflammatory scores up to moderate levels and minimal to mild Mayo scores.

\section{CONCLUSION}

The expression of Caspases- 4 and -5 within intestinal epithelial cells is highly specific biomarkers of colorectal carcinoma,regardless of whether it occurs following IBD or sporadically. Furthermore, they are a potential markers of dysplasia in IBD and colorectal polyps.

\section{REFERENCES}

1. Karim A (2018): Systematic Reviews and Meta-analytic Methods: Not All Are Created Equal. Diseases of the Colon \& Rectum, 61 (11): 1241-1242.

2. Abdu-Allah H, El-Shorbagi A, Abdel-Moty $\mathrm{S}$ et al. (2016): 5-Aminosalyclic Acid (5-ASA): A Unique AntiInflammatory Salicylate. Med chem (Los Angeles), 6: 5.

3. Gohar S, AlHassanin S, El-Assal M, and Hussein A (2015): Clinico-Epidemiology Study of Colorectal Cancer in Menofia University Oncology Department . Nature and Science, 13(11): :98-105.

4. Flood B, Oficjalska K, Laukens D et al. (2016): Altered expression of Caspases-4 and -5 during inflammatory bowel disease and colorectal cancer: diagnostic and therapeutic potential. Clinical and Experimental Immunology, 181: 39-50.

5. Terrinca J, Ana BR, Espino J et al. (2016): Extracellular heat shock proteins protect U937 cells from $\mathrm{H} 2 \mathrm{O} 2$ - induced apoptotic cell death. Mol Cell Biochem., 412: 19-26. 
6. Philchenkov A, Zavelevich M, Kroczak T et al. (2002): Caspases and cancer: mechanism of inactivation and new treatment modalities. Exp Oncol., 26 (2): 82-97.

7. Esmat S, El Nady M, Elfekki M et al. (2014): Epidemiological and clinical characteristics of inflammatory bowel diseases in Cairo, Egypt. World J Gastroenterol., 20 (3): 814-821.

8. Abou-Zeid A, Khafagy W, Marzouk D et al. (2017): Colorectal Cancer in Egypt. Diseases of the Colon \& Rectum, 45 (9): 1255-60 .

9. Ullman T, Odze R, Farraye FA (2009): Diagnosis and management of dysplasia in patients with ulcerative colitis and Crohn's disease of the colon. Inflamm. Bowel Dis., 9 (15): 630-8.

10.Ogino S, Nishihara R, Lochhead $P$ et al. (2012): Prospective Study of Family History and Colorectal Cancer Risk by Tumor LINE-1 Methylation Level. J Nation Cancer Instit., 5:1-11

11. Dabbous H, Mohamed Y, El-Folly $\mathrm{R}$ et al. (2018): Evaluation of Fecal M2PK as a Diagnostic Marker in Colorectal Cancer. Journal of Gastrointestinal Cancer, 1:9.

12. Kaitha S, Bashir $M$, and Ali $T$ (2015): Iron deficiency anemia in inflammatory bowel disease. World J Gastrointest Pathophysiol., 6 (3): 62-72.

13.Patel D, Trivedi C, Khan N (2018): Management of Anemia in Patients with Inflammatory Bowel Disease (IBD). Current Treatment Options in Gastroenterology, 16 (1): 112-128.

14. Mohamed $\mathrm{K}$, Ismail A, Hassana $\mathrm{E}$ et al. (2016): Efficacy and safety of infliximab on colonic mucosal healing in patients with moderate- to- severe ulcerative colitis. Journal of Current Medical Research and Practice, 2: $42-46$.

15. Ananthakrishnan A, Cheng S, Cai T et al. (2014): Serum Inflammatory Markers and Risk of Colorectal Cancer in Patients With Inflammatory Bowel Diseases. Clin Gastroenterol and Hepatol., 12: 1342-1348.

16. Vukobrat-Bijedic Z, Husic-Selimovic A, Sofic A et al. (2013): Cancer Antigens (CEA and CA 19-9) as Markers of Advanced Stage of Colorectal Carcinoma. Med Arh., 67: 397-401.

17. Yeo S, Chew M, Koh P, Tang C (2013): Young colorectal carcinoma patients do not have a poorer prognosis: a comparative review of 2,426 cases. Tech Coloproctol., 17: 653-661.
18.Metwally I, Shetiwy M, Elalfy A et al. (2017): Epidemiology and survival of colon cancer among Egyptians: a retrospective study. Journal of Coloproctology, 38 (1): 24-29.

19.Maher M, Nassar M (2009): Acute Cytomegalovirus Infection Is a Risk Factor in Refractory and Complicated Inflammatory Bowel Disease. Dig Dis Sci., 54: 24562462.

20.Moustafa A, Li W, Anderson A, Wong E et al. (2018): Genetic risk, dysbiosis, and treatment stratification using host genome and gut microbiome in inflammatory bowel disease. Clinical and Translational, 9: 132.

21.Davis EM, Kaufmann Y, Goyne $H$ et al. (2017): Pyroptosis of intestinal epithelial cells is crucial to the development of mucosal barrier dysfunction and intestinal inflammation. Gastroenterology, 152: S967.

22.Mathonnet M, Perraud A, Christou N et al. (2014): Hallmarks in colorectal cancer: angiogenesis and cancer stem-like cells. World J Gastroenterol., 20: 4189-96.

23.Lakshmanan, U, Porter AG (2007): Caspase-4 interacts with TNF receptorassociated factor 6 and mediates lipopolysaccharide-induced NF-kappaBdependent production of IL-8 and CC chemokine ligand 4 (macrophageinflammatory protein-1). J. Immunol., 179: 8480-8490.

24.Shibamoto M, Hirata H, Eguchi $H$ et al. (2017): The loss of CASP4 expression is associated with poor prognosis in esophageal squamous cell carcinoma. Oncology letter, 13: 1761-1766.

25.Lu H, Ouyang $W$ and Huang $C$ (2006): Inflammation, a key event in cancer development. Mol Cancer Res., 4: 221-33.

26.Smyth MJ, Cretney E, Kershaw MH, and Hayakawa $Y$ (2004): Cytokines in cancer immunity and immunotherapy. Immunol., 202: 275-93.

27.McSkeane D, Flood B, Fay J, Manils J et al. (2016): Altered expression of caspase-4 in colorectal polyps. The Irish Society of Gastoenterology (ISG); summer meeting 2016 . Available at: https://www.isge.ie/abstracts/alteredexpression-caspase-4-colorectal-polyps/

28.Oficjalska K, Raverdeau M, Aviello G et al. (2015): Protective role for Caspase-11 during acute experimental murine colitis. J Immunol., 194: 1252-60. 